Journal of Engineering and Applied Sciences 15 (2): 415-420, 2020

ISSN: 1816-949X

(C) Medwell Journals, 2020

\title{
Field Problems of Distance Relays with Combined Single/Double Circuit Transmission Lines
}

\author{
${ }^{1,2}$ Tamer A. Kawady, ${ }^{1,3}$ Gamal M.A. Sowilam and ${ }^{1}$ Raed A. Shalwala \\ ${ }^{1}$ Department of Electrical Engineering, Faculty of Engineering and Islamic Architecture, \\ Umm Al-Qura University, Mecca, Saudi Arabia \\ ${ }^{2}$ Department of Electrical Engineering, Faculty of Engineering, Minoufiya University, Minoufiya, Egypt \\ ${ }^{3}$ Department of Electrical Power and Machine Engineering, \\ Faculty of Engineering, Helwan University, Helwan, Egypt
}

\begin{abstract}
$\overline{\text { Abstract: Parallel transmission lines are characterized with a significant increase in the mutual coupling effects }}$ among the different phases of the coupled line segments raising remarkable errors for impedance-based protection equipment in particular. Owing to these situations, the total line impedance of each phase of the associated line may significantly change resulting from the mutually reflected impedances from the other phases. Thus, double line segments, when combined to single circuit ones, can significantly lead to have a deviated measured fault impedance from those parameters that are adjusted during the relaying setting stage. This may, accordingly, cause remarkable errors for distance protection algorithms in the field leading to unnecessary tripping or delayed fault clearing. In this study, the impact of the combination of single and double overhead line segments on the performance of distance relaying function is thoroughly investigated. This investigation is carried out using the Electro-Magnetic Transient Program (EMTP) using distributed parameter line modeling. The accuracy of the developed simulation is validated as compared with a recorded fault case for a $400 \mathrm{kV}$ combined single/double line from the field. This corroborated the correctness and the accuracy of the constructed model. The relay enabled functions and setting are collected and realized in the MATLAB environment. This research is essential for visualizing the core of the problems of these transmission networks with distance function and can consequently help to realize a practical and reliable distance relaying for such lines.
\end{abstract}

Key words: Parallel lines, distance relays, frequency dependent parameters, EMTP, MATLAB, networks

\section{INTRODUCTION}

Double circuit transmission lines are always characterized by a remarkable increase in their mutual coupling effects. This results in different problems particularly for protection equipment. For the distance relaying in particular, these effects remarkably increase the resulting estimation errors in the computed distance. Actually, when the parallel lines have similar parameters and configuration, the effects of mutual coupling are effectively cancelled as both lines will usually share the zero sequence currents due to the remote ground faults. However, for a fault on the line beyond the remote terminal end of a parallel line circuit, the distance relay will still under-reach for its zones setting. On the other hand, this balance of parameters and configuration is not the situation in most cases where tower mismatches as well as the environmental causes may increase the mutual impacts remarkably. Moreover, those hybrid transmission systems combining different line configurations have severe impacts on the distance relaying performance. These hybrid lines can combine different overhead-cable line segments or single-double circuit lines to be covered by the same distance relay. These situations are typically faced for some certain geographical or environmental circumstances including integrating different adjacent networks or passing through under-water cable segments. As a typical example for this line category is the $500 \mathrm{kV}$ hybrid line in Southern California (USA) comprising approximately as follows. The 33 miles of a single-circuit overhead line sharing a common tower structure with a $230 \mathrm{kV}$ line is followed by a 28 miles of a split-phase double-circuit overhead line on a double-circuit tower sharing the common tower structure and 4 miles of split-phase underground double circuit cables. Then, it is connected to a 8 miles of a single circuit overhead line with $500 \mathrm{kV}$ line. This complex configuration is subjected to varieties of technical challenges regarding their protection system as described by Bucco et al. (2017). Another example is the $400 \mathrm{kV}$ inter-tie Egypt-Jordan integration with both single and double circuit segments (Zahran et al., 2017). The mutual coupling is effectively measurable in all multiphase systems, in which the total line impedance significantly changes resulting from the

Corresponding Author: Tamer A. Kawady, Faculty of Engineering, Umm Al-Qura University, Makkah, Saudi Arabia Tel: +966559575046 
mutually reflected impedances from the other phases. Thus, the actual line parameters significantly deviate from those parameters that are adjusted for relay setting (Kawady and Stenzel, 2002, 2003). Moreover, the problem of mutual coupling is really not constant and strongly depends on different interacting factors such as conductor's spacing and voltage levels. On the other hand, common utilized mathematical cores of almost all distance relays in the field deals efficiently with uniform lines depending on simple RL Model in measuring the impedance between the relay and fault point. Thus, it is expected that remarkable errors arise with such relays when employed with these complex situations. Accordingly, the majority of publications in the literature are for ordinary lines considering uniform line configuration. However, more sophisticated and accurate line modeling and relaying mathematical cores are required for investigating such cases (Kasztenny et al., 2004).

As known, the distance relay operates when the measured impedance enters the adopted operation characteristics. The non-homogeneity of the overall line sections may result in inconsistent ratios of reactance to resistance and the zero sequence to the positive sequence impedances for each line segment. Thus, combining double circuit lines with single circuit segment or combining overhead line with cable segments together can influence the performance of the distance functions letting the fault be more distant from its actual point. These effects may consequently lead to unnecessary tripping of the local relay or to undesirable acceleration of the remote one (Zipp et al., 1997). Protective relaying unfortunately, the utilized protective functions with such hybrid lines are typically similar to those used for uniform lines. Hence, some certain recommendations should be issued during the setting stage of such protection functions in order to realize their proper setting profiles.
These recommendations may differ from one situation to another depending on its own parameters and circumstances. This complicates the process of their setting assessment and may need sometimes more trails. This study presents a visualization of this particular situation using both simulation and recorded fault cases from the field. The selected line is accurately modeled with a detailed distributed line model in EMTP for realizing a close representation of this line. The MATLAB is also utilized to carry out the required protection analysis study. The second section describes the selected line configuration and its accompanied protection system whereas its modeling details are described in third section Next one described the recorded test case from the field. Finally, simulation tests are investigated and analyzed.

\section{MATERIALS AND METHODS}

\section{Selected simulation system}

Main 400 kV system: A real $400 \mathrm{kV}, 750 \mathrm{MW}$ transmission system connecting two different networks are considered as a simulation example as described in Fig. 1. It comprises of a combination of a $33.6 \mathrm{~km}$ single circuit line and a $10 \mathrm{~km}$ overhead double circuit line. The single circuit line includes a $20 \mathrm{~km}$ overhead line and a $13.6 \mathrm{~km}$ of underground cable.

Modeling of the accompanied distance relays: Adopted distance relays for the $400 \mathrm{kV}$ hybrid transmission line are as given in Fig. 2 and 3. The adopted distance relays at both line ends have 4-zones using mho and quadrilateral characteristics for phase and ground faults, respectively. The related setting of the adjusted distance characteristics is described in Table 1. Each relay at both line ends are communicated together with Overreach Blocking (OB) with independent zone-1 as illustrated in Fig. 4.

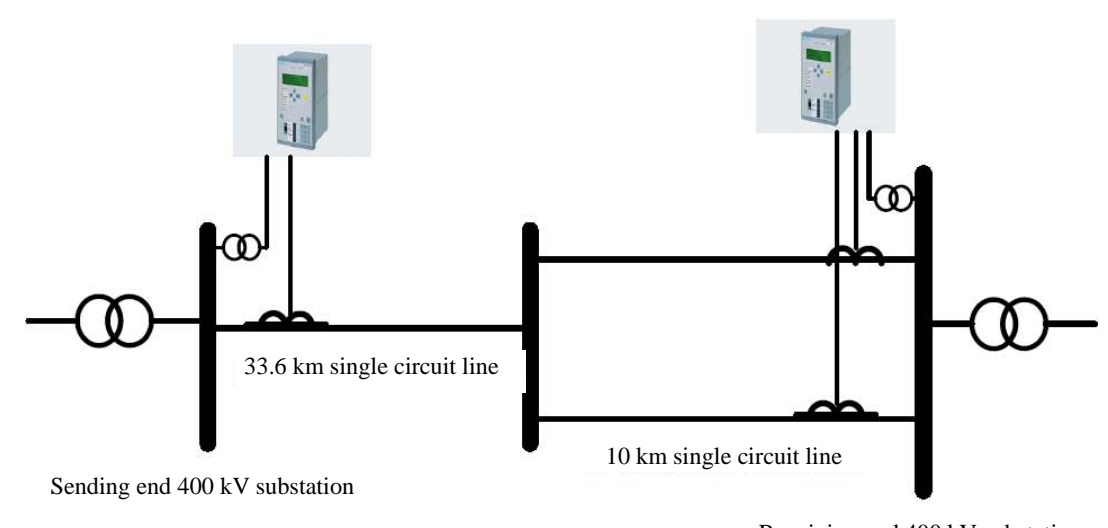

Receiving end $400 \mathrm{kV}$ substation

Fig. 1: Selected single/double circuit line from the fields 


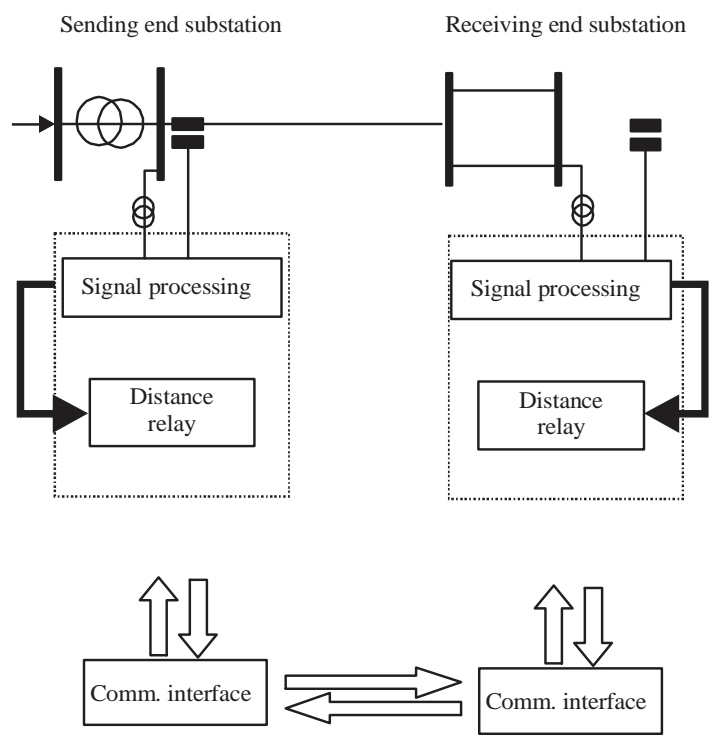

Fig. 2: Utilized protection system of the selected line
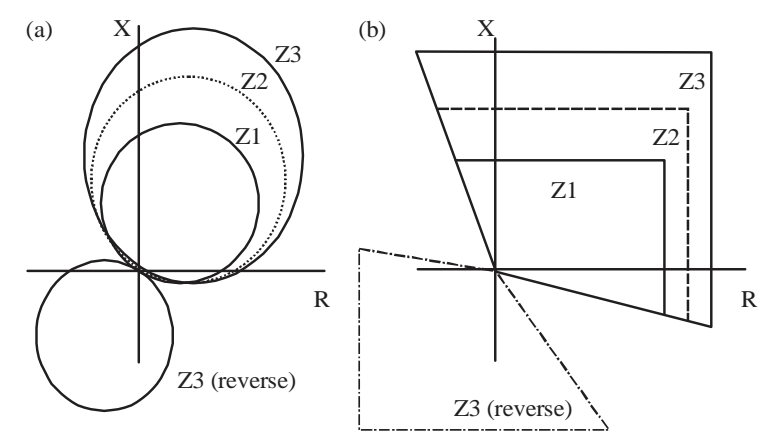

Fig. 3(a-b): Simulated settings for multi-zones distance relay (a) Mho characteristics for phase distance relays and (b) Quadrilateral characteristic for ground distance relays

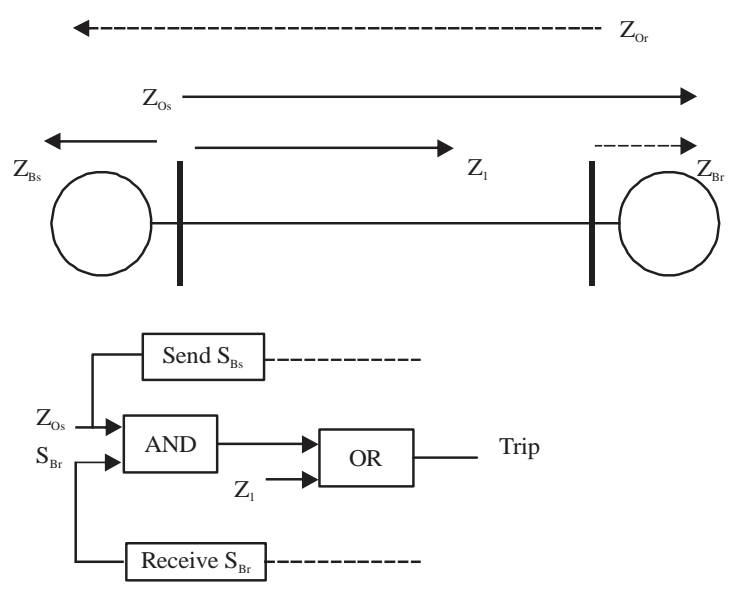

Fig. 4: Communication mode with blocking overreach with independent zone 1
Table 1: Selected setting for the distance relays

\begin{tabular}{lccc}
\hline $\begin{array}{l}\text { Relay } \\
\text { zone }\end{array}$ & Primary reach $(\Omega)$ & Secondary reach $(\Omega)$ & $\begin{array}{c}\text { Time } \\
\text { delay } \\
\left(\mathrm{m} \mathrm{sec}^{-1}\right)\end{array}$ \\
\hline Z1 & $1.8424+\mathrm{j} 7.02196$ & $0.4606+\mathrm{j} 1.75549$ & 0.0 \\
Z2 & $1.93913+\mathrm{j} 25.2915$ & $0.49229+\mathrm{j} 6.3228$ & 450 \\
Z3 & $1.93913+\mathrm{j} 41.96$ & $0.49229+\mathrm{j} 10.49$ & 795 \\
Z3 (reverse) & $1.93913+\mathrm{j} 41.96$ & $0.3446+\mathrm{j} 10.5088$ & 800 \\
\hline
\end{tabular}

Simulation development: The EMTP is a widely used package for simulating the electromagnetic transients for power system studies. Various models are available in the EMTP for each element in order to fulfill all application requirements and constraints. For our application in particular, the representation perspectives for each element of the system of Fig. 1 are described as follows (EPRI and EMTP-DCG, 1999).

Overhead line modeling: Different types of transmission line models are available in the EMTP starting from simple lumped parameter one to the distributed parameters modeling. Also, different options are available to account for the mutual coupling among the adjacent conductors. Among these models, the Frequency Dependent (FD-JMARTI) line Model arises as the most accurate one for simulation purposes. The FD-JMARTI line Model represents the true nature of a transmission line by modeling the frequency dependent and distributed line parameters. All single lines segments of Fig. 1 and double circuit line ones are modeled with the FD-JMARTI Model with the Line Constant Auxiliary routine of the EMTP depending on the actual tower configuration and conductor types (EPRI and EMTP-DCG, 1999). It is worthy to know that the first $5 \mathrm{~km}$ of overhead line segments are selected with AAAC conductor type $\left(400 \mathrm{~mm}^{2}\right)$ whereas the remaining lengths of the overhead line segments are selected with the ACSR conductor type $\left(490 / 65 \mathrm{~mm}^{2}\right)$.

Transformer modeling: Power Frequency Transformer Module (PFTM) in the EMTP is responsible to simulate the fundamental transformer model. This is available for normal and faulty transformer operation as long as the capacitance between windings and tank, windings and core and between winding layers can be ignored. Thus, the validity of this model is limited for frequency ranges from the power frequency to $10 \mathrm{kHz}$, depending on the transformer type (EPRI and EMTP-DCG, 1999). It is therefore, accurate enough for representing short circuit studies and relaying applications.

Underground cable modeling: Three single core cables are used for the 13.6 single circuit cable segment with $420 \mathrm{kV}$ were used with cupper cross section of $1000 \mathrm{~mm}^{2}$, conductor inside radius of 8.65 and $20.6 \mathrm{~mm}$ for the 
conductor outside radius. The EMTP provides different alternatives for cable modeling starting from a simple lumped model to sophisticated ones taking the parameter distribution and the frequency dependency of its parameters into account. Since, the system accuracy arises as an important issue, only distributed parameter cable is considered with the FDLMARTI Model. It considers the distribution of all cable parameters as well as the frequency dependence of the transformation matrix elements. It arises as the most accurate and efficient cable model in the EMTP (Tavares et al., 1999; Marti, 1988, 1993). Therefore, it is employed for developing the related parameters of the submarine cable section.

Protection scheme modeling: Figure 3 shows the adopted setting of the 4-zones distance relays at both line ends relays with mho and quadrilateral characteristics respectively with the parameters shown in Table 1 . Each relay at both line ends are communicated together with Overreach Blocking (OB) with independent zone-1. In spite of the superior performance of the EMTP for modeling power system elements as well as some control structures, its ability for modeling large-scale control systems or sophisticated protection schemes is relatively limited. This is mainly attributed to the poor mathematical manipulation offered, especially, for those modern digital protection and control schemes evolving advanced mathematics. The MATLAB is however, more distinctive with its ultimate mathematical and logic abilities. Thus, it provides a candidate for developing accurate representation of modern digital protection schemes. Then, collaboration between both simulation tools is adopted for studying and analyzing the performance of these systems.

Recorded fault case: Typically, fault cases are recorded by utilizing Hathaway-Digital Fault Recorder (DFR) running at both the sending end and receiving end of the selected test system. These test cases facilitate validating the constructed models as well analyzing the performance of the corresponding protective relays for various faulty abnormal conditions. A line to ground fault occurred beyond the receiving end transformer during a $150 \mathrm{MW}$ transferred load from the sending end to the receiving end. The recorded case is shown in Fig. 5 where the three phase voltages are plotted with channels range 9-11 where the three phase currents are connected to channels 13-15. The fault has been classified successfully in the reverse zone (zone-4) as the fault occurred just behind the distance relay at the receiving end substation. Then, tripping of the local breaker at the receiving end substation was inhibited. Also, a blocking signal was

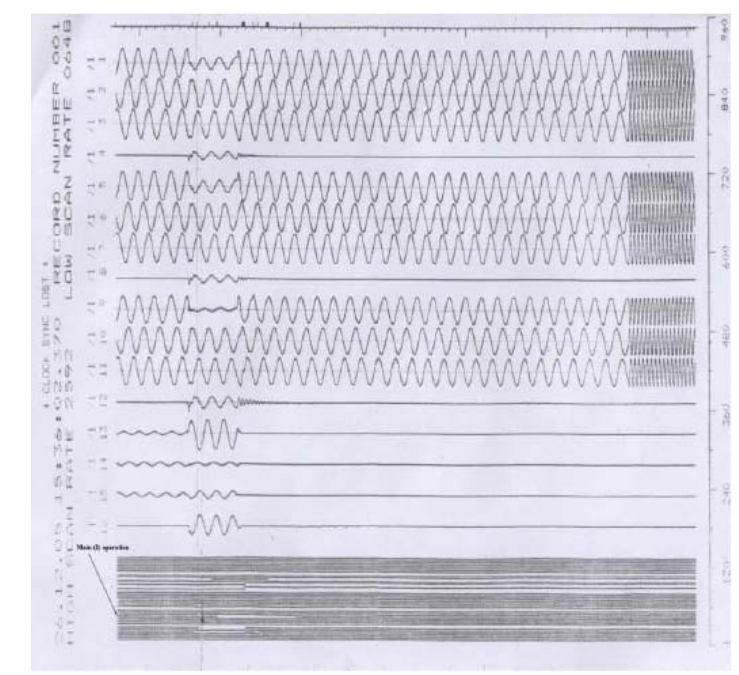

Fig. 5: Field recorded test cases using the digital fault recorder type HATHAWAY for a single line-to-ground with $150 \mathrm{MW}$ pre-fault loading

initiated for the corresponding relay at the sending end substation. In spite of the received blocking signal, an accelerated tripping was surprisingly initiated resulting from the zone1-independency setting mode. This optional mode of setting enables the distance element to initiate an instantaneous tripping regardless the communication status. The event binary status for the corresponding relay was illustrated Fig. 5 by the binary event channel of 28 .

\section{RESULTS AND DISCUSSION}

\section{Simulated testing}

Verifying the recorded test case: There is no doubt that comparing the performance of the developed model with some recorded field data, if available is a trustful method for fulfilling this target. Nowadays, modern multi-functions Digital Fault Recorders (DFRs) are usually installed in the substations and power plants. The DFR's enable data logging of vital events and fault oscillography records. The value of these real field cases is obvious for the validation of the system models and addressing problems that may exist. Fortunately, different relevant fault cases to the addressed problem have been recently recorded. Among these test cases, the described one was recorded as seen in the preceding section. Figure 6 show the EMTP simulated response of the sending end distance relay for the recorded line to ground fault occurring beyond the receiving end transformer with a 150 MW transferred load. As illustrated form the aforementioned figure, the estimated fault impedance to the fault point drop below zon-1 setting. Note that, the impedances are calculated using the voltage and current 


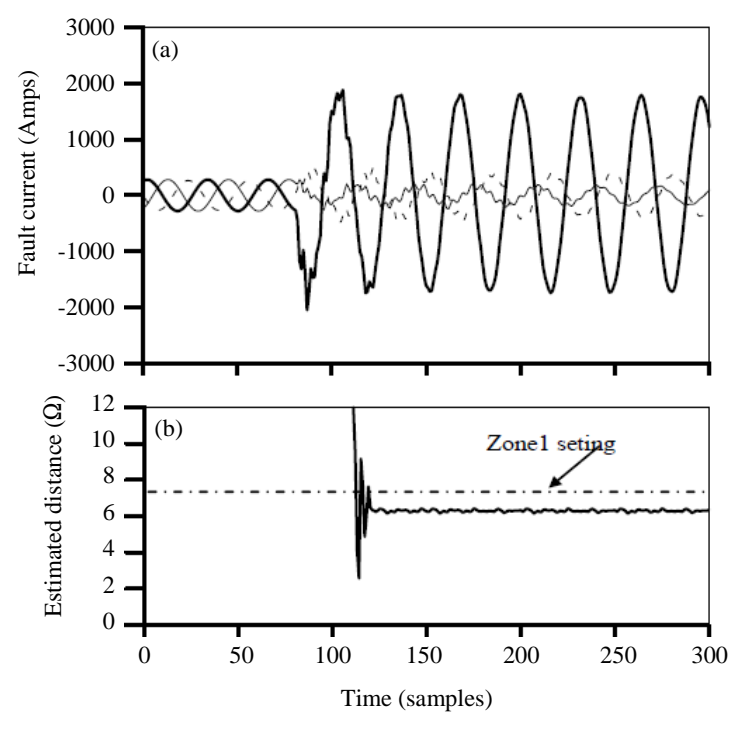

Fig. 6(a-b): Simulated response of the sending end distance relay to the recorded fault case beyond the receiving end transformer

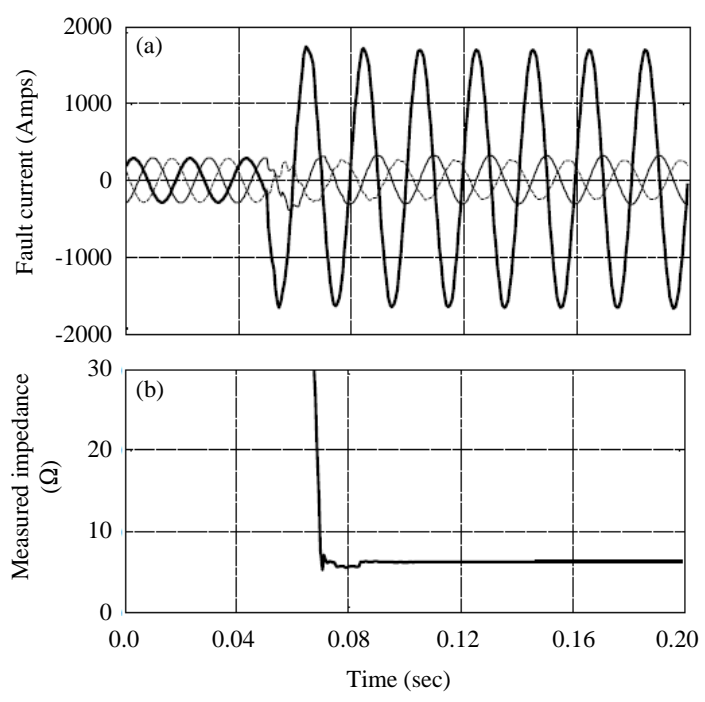

Fig. 7(a-b): Scheme response evaluation for a single phase to ground fault at $20 \mathrm{~km}$ from the sending end (a) Fault current and (b) Estimated fault distance

phasor quantities. These phasors are extracted using Optimized Parallel Discrete Fourier Transform (OPDFT). This orthogonal filter has been recently introduced to the area of numerical relaying. OPDFT combines accuracy, low computation time and stable output for both fixed and float point CPU's (Darwish and Fikri, 2007). This is because accurate signal processing tools is essential for realizing a precise relaying performance. The results

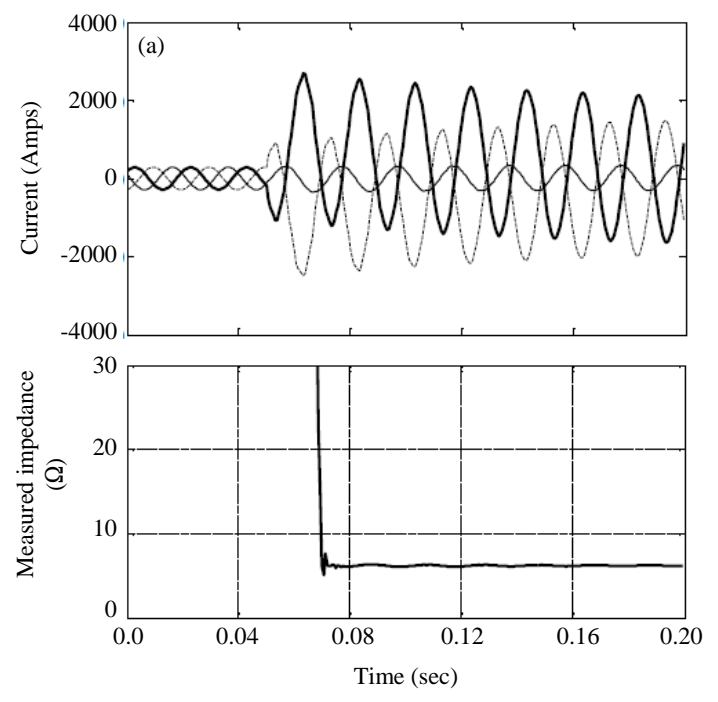

Fig. 8(a-b): Scheme response evaluation for a double phase fault at $20 \mathrm{~km}$ from the ending end (a) Fault current and (b) Estimated fault distance

show acceptable estimated impedances for both fault conditions as compared with the recorded one with estimated errors below $5 \%$ of the segment line length.

Simulated transient behavior of the distance relay: Testing certain relaying functions such as the distance relaying at some selected fault points may emphasize the model accuracy. This can be carried out by comparing the computed distances by the relays with their actual fault distances. Lower errors of the estimated fault impedance as compared with the actual $s$ reveal the accuracy grade of the developed model. Figure 7 and 8 show the estimated fault distances for line-to-ground and line-to-line faults occurring at $20 \mathrm{~km}$ from the sending end, respectively. The results corroborated the accuracy of the developed model with estimation error of $3 \%$ of the segment line length only.

\section{CONCLUSION}

In this study, the performance of the distance relaying with hybrid transmission systems was investigated. For faults located at the homogeneous part of the line, the distance relay reacted correctly, since, the double circuit part or the cable segment were not involved into the faulted equivalent circuit seen by the relay. However, higher percentages of estimated distance errors resulted for similar faults located involving the nonhomogeneous part of the line as indicated for the fault occurring in zone 2 (beyond the double circuit part where it was recognized as a zone1 fault by the sending end relay. It surprisingly neglected the blocking signal sent from the 
receiving end relay. It can be therefore, concluded that the selected communication mode played a role for inhibiting or permitting the false tripping. It should be, therefore, carefully selected and adjusted.

\section{ACKNOWLEDGEMENTS}

The researchers would like to thank Deanship of Scientific Research at Umm Al-Qura University (UQU), Saudi Arabia for the financial Support of this work (Project ID: 43408008).

\section{REFERENCES}

Bucco, D., C. Sanden, A. Torres, E. Wong and J. Bell et al., 2017. Protection challenges for North Americas first combined cable/overhead double-circuit $500 \mathrm{kV}$ transmission line with mutual coupling. Proceedings of the 2017 70th Annual International Conference on Protective Relay Engineers (CPRE), April 3-6, 2017, IEEE, College Station, Texas, USA., ISBN: 978-1-5386-1582-9, pp: 1-15.

Darwish, H.A. and M. Fikri, 2007. Practical considerations for recursive DFT implementation in numerical relays. IEEE. Trans. Power Delivery, 22: 42-49.

EPRI and EMTP-DCG., 1999. Electromagnetic transient programs (EMTP96) rule book-Ver. Development Coordination Group (DCG), Electric Power Research Institute, Palo Alto, California, USA.

Kasztenny, B., I. Voloh and J.G. Hubertus, 2004. Applying distance protection to cable circuits. Proceedings of the 57th Annual International Conference on Protective Relay Engineers, April 1, 2004, IEEE, College Station, Texas, USA., ISBN: 0-7803-8432-6, pp: 46-69.
Kawady, T. and J. Stenzel, 2002. Investigation of practical problems for digital fault location algorithms based on EMTP simulation. Proceedings of the IEEE/PES Asia Pacific Conference on Transmission and Distribution Exhibition Vol. 1, October 6-10, 2002, IEEE, Yokohama, Japan, ISBN:0-7803-7525-4, pp: 118-123.

Kawady, T. and J. Stenzel, 2003. A practical fault location approach for double circuit transmission lines using single end data. IEEE. Trans. Power Delivery, 18: 1166-1173.

Marti, L., 1988. Simulation of transients in underground cables with frequency-dependent modal transformation matrices. IEEE. Trans. Power Delivery, 3: 1099-1110.

Marti, L., 1993. Simulation of electromagnetic transients in underground cables using the EMTP. Proceedings of the 1993 2nd International Conference on Advances in Power System Control, Operation and Management (APSCOM-93), December 7-10, 1993, IET, Hong Kong, ISBN:0-85296-569-9, pp: 147-152.

Tavares, M.C., J. Pissolato and C.M. Portela, 1999. Guidelines for transmission line modeling. Proceedings of the 1999 IEEE International Conference on Power Engineering Society Summer Meeting (Cat. No. 99CH36364) Vol. 2, July 18-22, 1999, IEEE, Edmonton, Alta, Canada, pp: 786-791.

Zahran, A.D., N.I. Elkalashy, M.A. Elsadd, T.A. Kawady and A.M.I. Taalab, 2017. Improved ground distance protection for cascaded overhead-submarine cable transmission system. Proceedings of the 2017 9th International Conference on Middle East Power Systems (MEPCON), December 19-21, 2017, IEEE, Cairo, Egypt, ISBN: 978-1-5386-0991-0, pp: 778-758.

Zipp, J., M. Conroy, K. Behrendt, T. Gallen and I. Hasenwinkle et al., 1997. Protective relaying considerations for transmission lines with high voltage AC cables. IEEE. Trans. Power Delivery, 12: 83-96. 